\title{
Inverse Association between Serotonin 2A Receptor Antagonist Medication Use and Mortality in Severe COVID-19 Infection
}

\author{
Mark B. Zimering ${ }^{1,2 *}$, Tanzila Razzaki ${ }^{1,3}$, Tiffany Tsang ${ }^{1,3}$ and John J. Shin ${ }^{1,3}$ \\ ${ }^{1}$ Medical Service, Veterans Affairs New Jersey Healthcare System, East Orange, New Jersey, USA \\ ${ }^{2}$ Rutgers-Robert Wood Johnson Medical School, New Brunswick, NJ, USA \\ ${ }^{3}$ Rutgers-New Jersey Medical School, Newark, New Jersey, USA
}

${ }^{*}$ Corresponding author: Dr. Mark B. Zimering, MD, PhD. Chief, Endocrinology, Veterans Affairs New Jersey Healthcare System, 385 Tremont Ave, East Orange, New Jersey 07018, USA; Tel: 9086470180 x4426; Fax: 908 604-5249; E-mail: mark.zimering@va.gov

Received: September 05, 2020; Accepted: September 10, 2020; Published: September 15, 2020

\begin{abstract}
Advanced age and medical co-morbidity are strong predictors of mortality in COVID-19 infection. Yet few studies (to date) have specifically addressed risk factors associated with COVID-19 mortality in a high-risk subgroup of older US adults having one or more chronic diseases. Our hypothesis is that medications having 'off-target' anti-inflammatory effects may play a role in modulating the immune response in COVID-19 infection. We analyzed baseline risk factors associated with respiratory failure or death in 55 older adult US military veterans hospitalized for COVID-19 infection during (March-June 2020) the peak of the pandemic in New Jersey. Fifty-three percent (29/55) of patients experienced respiratory failure and thirty-one percent $(17 / 55)$ died. In adjusted logistic regression analysis, baseline neutrophil to lymphocyte ratio (NLR) $(\mathrm{P}=0.0035)$ and body mass index $(P=0.03)$ were significant predictors of the risk for respiratory failure. Age $(P=0.05)$ and non-use (vs. use) of psychotropic medications having serotonin $2 \mathrm{~A}$ receptor antagonist properties (odds ratio 5.06; 95\% confidence intervals 1.18-21.7; $\mathrm{P}=0.029$ ) was each a significant predictor of an increased risk of death. There was a significant interaction effect of age and non-use (vs.. use) of psychotropic serotonin $2 \mathrm{~A}$ receptor antagonist medications on the odds ratio $(\mathrm{OR})$ for death $(P=0.011)$. In selected, ventilator-dependent COVID-19 pneumonia patients treated with psychotropic serotonin 2A receptor antagonist medications to control agitation and ICU delirium, there was an apparent positive association between medication use and significant rise in the absolute lymphocyte count and decrease in the neutrophil: lymphocyte ratio. Taken together, these data are the first to suggest that certain psychotropic medications used in the treatment of chronic psychiatric illness and/or for acute delirium are inversely associated with mortality in severe COVID-19 infection by unknown mechanism which may involve (in part) immunomodulatory effects.
\end{abstract}

\section{Introduction}

According to the Centers for Disease Control [1], medical co-morbidity was associated with a substantially increased risk of admissions to the ICU due to severe COVID-19 infection. Hypertension, diabetes and advanced age were among the risk factors associated with increased mortality due to severe COVID-19 infection in a recent large study from metropolitan New York [2]. Older adult U.S. military veterans carry a substantial burden of medical comorbidities including type 2 diabetes mellitus and hypertension [3]. Yet few studies to date have examined risk factors predictive of severe COVID-19 infection in patients having one or more chronic illnesses. The aim of the present study was to evaluate risk factors associated with poor outcome in COVID-19 infection requiring hospitalization in older adult US military veterans. The SARS-Cov-2 virus mediates hyper-inflammation and dysregulated immunity leading to 'cytokine storm' [4]. Inflammation predisposes to hypercoagulability and platelet-derived serotonin (5-HT) promotes neutrophil infiltration at sites of injury, each process is recognized as a 'bad actor' in severe COVID-19 infection [5]. The 5-hydroxytryptamine $2 \mathrm{~A}$ receptor is expressed on platelets, innate and adaptive immune cells [6,7] and it was reported to 'drive' chronic inflammation in animal models of autoimmune diseases [8,9]. A secondary aim of the present study was to test whether psychotropic medications belonging (in part) to the class of 5-hydroxytryptamine $2 \mathrm{~A}$ receptor antagonists (in current use as anti-depressant or atypical antipsychotic medications) may modify the risk of death in severe COVID-19 infection.

\section{Patients and Methods}

The retrospective study was reviewed and approved by the local Veterans Affairs New Jersey Healthcare System Institutional Review Board. Patients were consecutively selected from among those inpatients admitted to an acute medical ward or intensive care unit (ICU) at the Veterans Affairs New Jersey Healthcare System (VANJHCS), East Orange campus between late March 2020 and early June 2020, i.e. during the peak of the pandemic in New Jersey. Nearly all patients tested COVID-positive $(n=53)$ by polymerase chain reaction of an oropharyngeal or nasopharyngeal swab. Two patients had a clinical picture consistent with COVID-19 pneumonia, but a negative COVID-19 PCR test and were included in the analysis. 
Ninety-six percent of patients were men. Forty-one of 55 patients (75\%) had respiratory symptoms on admission. Thirty-four percent of patients were 74 years or older, nearly all patients had at least one co-morbidity, including forty percent of patients who had a baseline history of cardiovascular disease (Table 1).

\section{Endpoint(s)}

Twenty-nine of fifty-five patients (52.7\%) experienced respiratory failure and there were 17 deaths which generally occurred within 2-3 months of the acute COVID-19 hospitalization. Unless a patient was readmitted to the same institution (VANJHCS), or had subsequent entries in the VANJHCS medical record, he or she was presumed to have survived acute episode of COVID-19 infection following his or her successful discharge home or to a lower-intensity, rehabilitativetype care facility.

Respiratory failure is defined as requiring intubation and mechanical ventilation or high-flow, concentrated oxygen, e.g. $50 \%$ oxygen via nasal cannula at 15 liters/min or $\geq 50 \%$ oxygen via a nonrebreather mask.

Diabetic microvascular complications - retinopathy - macular edema, or proliferative retinopathy, nephropathy - $>300 \mathrm{mg} / \mathrm{g}$ creatinine albuminuria; painful neuropathy - evidenced by treatment with gabapentin and clinical diagnosis determined by trained neurology staff. Neutrophil/Lymphocyte ratio (NLR) - is the average on two consecutive days of the absolute neutrophil count/absolute lymphocyte count which occurred at the nadir of the absolute lymphocyte count.

Clinical and other laboratory data was extracted from retrospective chart reviews. Body weight index (BMI), glycosylated hemoglobin value was based on prior results closest to the date of inpatient admission. Baseline insulin use or the use of angiotensin converting enzyme inhibitor or angiotensin receptor blocker medications was determined from baseline outpatient medication lists.

Table 1: Baseline clinical characteristics in the study patients.

\begin{tabular}{|l|c|}
\hline Risk Factor & Mean (SD) \\
\hline Age (years) & $70.6(11.5)$ \\
\hline BMI (kg/m $\left.{ }^{2}\right)$ & $29.0(7.5)$ \\
\hline Race AA/NHW/H (\%) & $47 / 35 / 11$ \\
\hline Co-morbidities & $(\%)$ \\
\hline Hypertension (\%) & 71 \\
\hline Diabetes (\%) & 58 \\
\hline Cardiac disease (\%). & 40 \\
\hline COPD/Asthma (\%) & 20 \\
\hline ESRD (\%) & 17 \\
\hline Psychiatric illness (\%) & 20 \\
\hline Medications/Treatments & $(\%)$ \\
\hline Psychotropic having Serotonin 2A receptor Antagonist activity (\%) & 45 \\
\hline ACEi/ARB (\%) & 44 \\
\hline Glucocorticoids (\%) & 33 \\
\hline Insulin (\%) & 25 \\
\hline Remdesivir (\%) & 5 \\
\hline Convalescent plasma (\%) & 2 \\
\hline
\end{tabular}

$\mathrm{N}=55$ patients; BMI: Body Mass Index; ACEi: Angiotensin Converting Enzyme Inhibitor; ARB: Angiotensin Receptor Blocker Medication; AA: African-American, NHW: NonHispanic White, H: Hispanic; COPD: Chronic Obstructive Pulmonary Disease; ESRD: End Stage Renal Disease.

\section{Patients Treated with Antipsychotic or Antidepressant Medications}

Treatment with an atypical, second-generation antipsychotic medication (SGA) or an antidepressant medication that has antagonist activity at the 5-hydroxytryptamine (serotonin)2A receptor was evaluated as a possible risk factor for the outcome of death vs. survival after acute COVID-19 infection. Both atypical antipsychotics and certain anti-depressants (tricyclic antidepressants, trazadone, mirtazapine) share high-affinity antagonism on the 5-hydroxytryptamine $2 \mathrm{~A}$ receptor. Total twenty patients were treated with a SAG antipsychotic medication (risperidone, olanzapine, quetiapine, aripiprazole) and five were treated with one of the 5-HT2AR antagonist antidepressant medications (trazadone, mirtazapine) either for an underlying mental health disorder (schizophrenia, schizoaffective disorder, major depressive disorder) or to manage acute agitation/delirium.

\section{Statistics}

Logistic regression analysis was used to model possible baseline risks factors associated with the occurrence of respiratory failure or death. Age and body mass index were included as covariates in the model when testing for an effect of other risk variables. In univariate regression analysis, other risk variables [NLR, diabetes mellitus, baseline use of various medication classes, baseline cardiac disease] were tested for association with the endpoint, and were included in the model if it reached 0.05 of significance level. Two way-interactions were checked among significant risk variables. Logistic regression was conducted using SAS 9.4 (SAS Institute Inc, Cary, NC).

\section{Results}

The baseline clinical characteristics in the study patients are shown in Table 1 . The mean age was $70.6 \pm 11.5$ years old. More than half of the patients had diabetes mellitus (nearly all type $2 \mathrm{DM}$ ) and one in four patients were treated with insulin. Mean glycosylated hemoglobin was $7.7 \pm 2.0 \%$ among diabetic patients and one-third had at least one microvascular complication (not shown in Table 1).

Lymphopenia is a characteristic laboratory feature in severe COVID-19 disease. The neutrophil to lymphocyte ratio (NLR) was reported to be a prognostic marker in COVID-19 infection: a threshold NLR value $>5.0 v s . \leq 5.0$ demonstrated high sensitivity and specificity for differentiating between severe and mild COVID-19 infection [10]. In univariate regression analysis, the neutrophil: lymphocyte ratio (Odds ratio: 1.232; 95\% confidence intervals 1.079-1.406; $\mathrm{P}=0.002$ ) was a significant predictor of the risk for respiratory failure (Table $2 \mathrm{~A}$ ).

In multivariate logistic regression that adjusted for age and body mass index, baseline NLR (OR 1.2554; 95\% CI: 1.0776-1.4624; $\mathrm{P}=0.0035)$ and BMI (OR 1.1429; 95\% CI: 1.0131-1.12893; $\mathrm{P}=0.030$ ) was each a significant predictor of the risk of respiratory failure in COVID-19 infection (Table 2B).

We next evaluated risk predictors of COVID-19 mortality. In univariate logistic regression analysis, the use of an antipsychotic or antidepressant medication having 5-HT2A receptor antagonist 
Table 2: Univariate (A) and multivariate (B) logistic regression of risk factors associated with Covid-19 respiratory failure.

\begin{tabular}{|l|c|c|c|}
\hline Variable & Odds Ratio & $\mathbf{9 5 \%}$ CI & P-value \\
\hline NLR & 1.232 & $1.079-1.406$ & 0.002 \\
\hline Age (years) & 1.039 & $0.355-3.038$ & 0.944 \\
\hline BMI $\left(\mathrm{kg} / \mathrm{m}^{2}\right)$ & 1.081 & $0.991-1.179$ & 0.231 \\
\hline Diabetes (yes/no) & 1.031 & $0.983-1.081$ & 0.212 \\
\hline Insulin (yes/no) & 1.890 & $0.540-6.617$ & 0.319 \\
\hline ACEi/ARB (yes/no) & 1.108 & $0.381-3.224$ & 0.851 \\
\hline
\end{tabular}

N=55; NLR: Neutrophil to Lymphocyte Ratio; CI: Confidence Interval.

B

\begin{tabular}{|l|c|c|c|}
\hline Variable & Odds Ratio & 95\% CI & P-value \\
\hline NLR & 1.255 & $1.078-1.462$ & 0.0035 \\
\hline BMI $\left(\mathrm{kg} / \mathrm{m}^{2}\right)$ & 1.143 & $1.013-1.129$ & 0.030 \\
\hline Age (years) & 1.052 & $0.993-1.134$ & 0.079 \\
\hline
\end{tabular}

N=55; NLR: Neutrophil to Lymphocyte Ratio; CI: Confidence Interval.

Table 3: Univariate logistic regression analysis of risk factors associated with Covid-19 death.

\begin{tabular}{|l|l|l|l|}
\hline Variable & Odds Ratio & $\mathbf{9 5 \% ~ C I ~}$ & P-value \\
\hline $\begin{array}{l}\text { Serotonin 2A R } \\
\text { Blocker med }\end{array}$ (yes/no). & & & \\
\hline Age (years) & 0.156 & $0.038-0.634$ & 0.0094 \\
\hline Cardiac disease (yes/no) & 1.076 & $1.015-1.141$ & 0.014 \\
\hline NLR & 3.095 & $0.948-10.109$ & 0.061 \\
\hline African-Am vs. other race or ethnicity & 1.031 & $0.992-1.072$ & 0.125 \\
\hline Diabetes (yes/no) & 0.700 & $0.220-2.225$ & 0.546 \\
\hline Glucocorticoids (yes/no) & 0.734 & $0.232-2.350$ & 0.599 \\
\hline Acei/ARB (yes/no) & 2.489 & $0.753-8.223$ & 0.135 \\
\hline Insulin (yes/no) & 0.606 & $0.186-1.975$ & 0.406 \\
\hline BMI (kg/m $\left.{ }^{2}\right)$ & 0.289 & $0.057-1.469$ & 0.101 \\
\hline
\end{tabular}

BMI: Body Mass Index; dz: Disease; ${ }^{\star}$ Second generation atypical antipsychotics, tricyclic antidepressants, trazadone or mirtazapine; CI: Confidence Interval, $\mathrm{N}=55$ patients.

properties (OR 0.198; 95\% CI:0.038-0.634; $\mathrm{P}=0.0094$ ) was a significant predictor of a decreased risk of death (Table 3). Age (OR 1.076; 95\% CI 1.015-1.141; $\mathrm{P}=0.0139)$ was a significant predictor of an increased risk of death (Table 3). Baseline cardiac disease (OR 3.095; 95\% CI 0.948$10.109 ; \mathrm{P}=0.0613$ ) was a nearly significantly predictive of an increased risk of death (Table 3 ).

In multi-variate logistic regression analysis, non-use of antipsychotic or antidepressant medications having 5-HT2AR antagonist properties (OR 5.058; 95\% CI: 1.180-21.689; $\mathrm{P}=0.029$ ) and age (OR 1.062; 95\% CI: 1.000-1.128; $\mathrm{P}=0.050$ ) was each a significant predictor of an increased risk of death in COVID-19 infection (Table 4A).

There was a significant interaction effect of age and 5-HT2A receptor-blocking classes of medication on the risk of death in COVID-19 infection ( $\mathrm{P}=0.0112$ ) (not shown in Table 4). Use of 5-HT2A receptor blocking medications was associated with low mortality rate $(0 / 18)$ in younger patients $(\leq 72$ years old) and the

Table 4: Multivariate logistic regression analysis of risk factors associated with Covid-19 death.

\begin{tabular}{|l|c|c|c|}
\hline Variable & Odds Ratio & 95\% CI & P-value \\
\hline $\begin{array}{l}\text { Serotonin 2A R } \\
\text { Blocker } \text { med }^{*} \text { (no/yes) }\end{array}$ & 5.058 & $1.180-21.689$ & 0.029 \\
\hline Age (years) & 1.062 & $1.000-1.128$ & 0.050 \\
\hline
\end{tabular}

$\mathrm{N}=55$ patients; ${ }^{*}$ Second generation atypical antipsychotics, tricyclic antidepressants, trazadone or mirtazapine; CI: Confidence Interval.

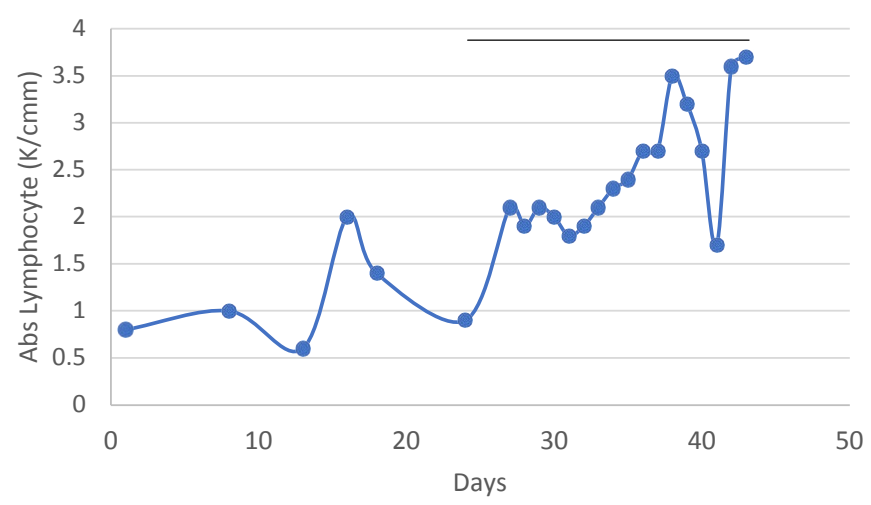

Figure 1: A 59-year old woman with systemic lupus erythematosus who developed hypoxemic respiratory failure. Solid line indicates nightly treatment with an oral secondgeneration anti-psychotic medication. The absolute lymphocyte count increased from 0.9 to $3.7 \mathrm{~K} / \mathrm{cmm}$ in association with SGA treatment. The patient was discharged to a rehabilitation center in stable condition.

overall mortality rate (3/8) increased among patients aged 73-95 years old treated with a 5-HT2A receptor blocking class of medication (not shown in Table 4).

In representative patients having hypoxemic respiratory failure, treatment with psychotropic medications having 5 -HT2A receptor blocking activity (to control agitation and/or delirium on the ventilator in the ICU) was associated with an abrupt increase in the absolute lymphocyte count and a decrease in the NLR.

\section{Case 1}

A 59-year old female with systemic lupus erythematosus developed hypoxemic respiratory failure. She manifested a high level of systemic inflammation, an initial C-reactive protein level > $320 \mathrm{mg} / \mathrm{L}$, normal (1-10) and 2 weeks later it was still $189 \mathrm{mg} / \mathrm{L}$. She was treated for 3 weeks with a nightly dose of an oral SGA to manage agitation/ delirium on the ventilator in the ICU. Her absolute lymphocyte count increased from 0.9 to $3.7 \mathrm{~K} / \mathrm{cm}^{2}$ in association with SGA treatment (solid line, Figure 1). She was discharged to a rehabilitation center in stable condition.

\section{Case 2}

A 55-year old man with T2DM experienced hypoxemic respiratory failure. The initiation of nightly atypical antipsychotic medication was associated with an abrupt rise in absolute lymphocyte count (solid bar, Figure $2 \mathrm{~A}$ ). The absolute lymphocyte count was significantly higher (Figure 2B) and the NLR was significantly lower (Figure 2C) during 7 days on SGA treatment compared to corresponding mean level 4 days before initiation of SGA treatment.

\section{Case 3}

A 76-year old man with T2DM and polyneuropathy developed hypoxemic respiratory failure and was intubated. During the initial 15 days he was treated with a combination of D2 receptor blockers (Haldol) and benzodiazepines to control agitation/delirium in the ICU on the ventilator (dashed line, Figure 3A). Three days after successful extubation (arrow, Figure 3A) the drug regimen was changed and he received an 18-day course of an oral SGA medication nightly to control agitation/delirium (solid line, Figure $3 \mathrm{~A}$ ). He was 


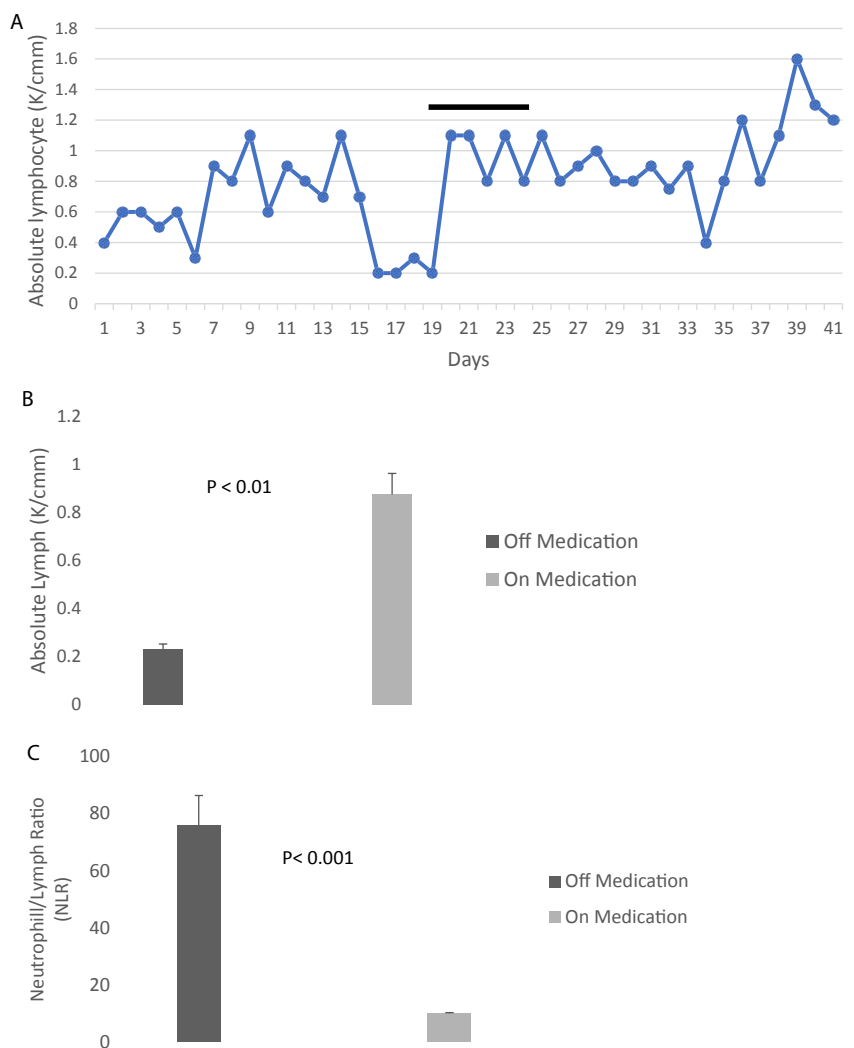

Figure 2: A 55-year old man with type 2 diabetes mellitus (T2DM) who developed hypoxemic respiratory failure. A) The initiation of treatment with nightly atypical, secondgeneration antipsychotic medication was associated with an abrupt rise in absolute lymphocyte count (solid bar). B) The absolute lymphocyte count was significantly higher and C) the NLR was significantly lower during 7 days on SGA treatment (Medication) compared to corresponding mean level on 4 consecutive days before initiation of SGA treatment (Medication).

later discharged (in stable condition) from the hospital. The mean absolute lymphocyte count was significantly higher during a 16-day period on daily treatment with an SGA compared to the mean level during a comparable, preceding 16-day period on intermittent D2 receptor antagonist and/or benzodiazepine medications (Figure 3B).

The mean neutrophil/lymphocyte ratio (NLR) was significantly lower (6.6 vs. 18.0; $\mathrm{P}<0.001)$ during the period on treatment with an SGA (atypical antipsychotic medication) compared to the corresponding preceding period on D2R antagonist and/or benzodiazepine medications to control agitation and delirium (Figure 3C).

\section{Discussion}

Biomarkers that predict an increased risk of severe COVID-19 infection can help guide therapy in selected patients. The present finding that the baseline neutrophil to lymphocyte ratio was a significant predictor of the risk for respiratory failure in older adult hospitalized patients suffering with COVID-19 pneumonia is consistent with other reports of the prognostic value of the NLR [10]. Yet, to our knowledge, this is the first report that treatment with certain psychotropic medications having 5-HT2A receptor blocking properties was associated with substantially lower mortality in severe COVID-19 infection. The apparent association
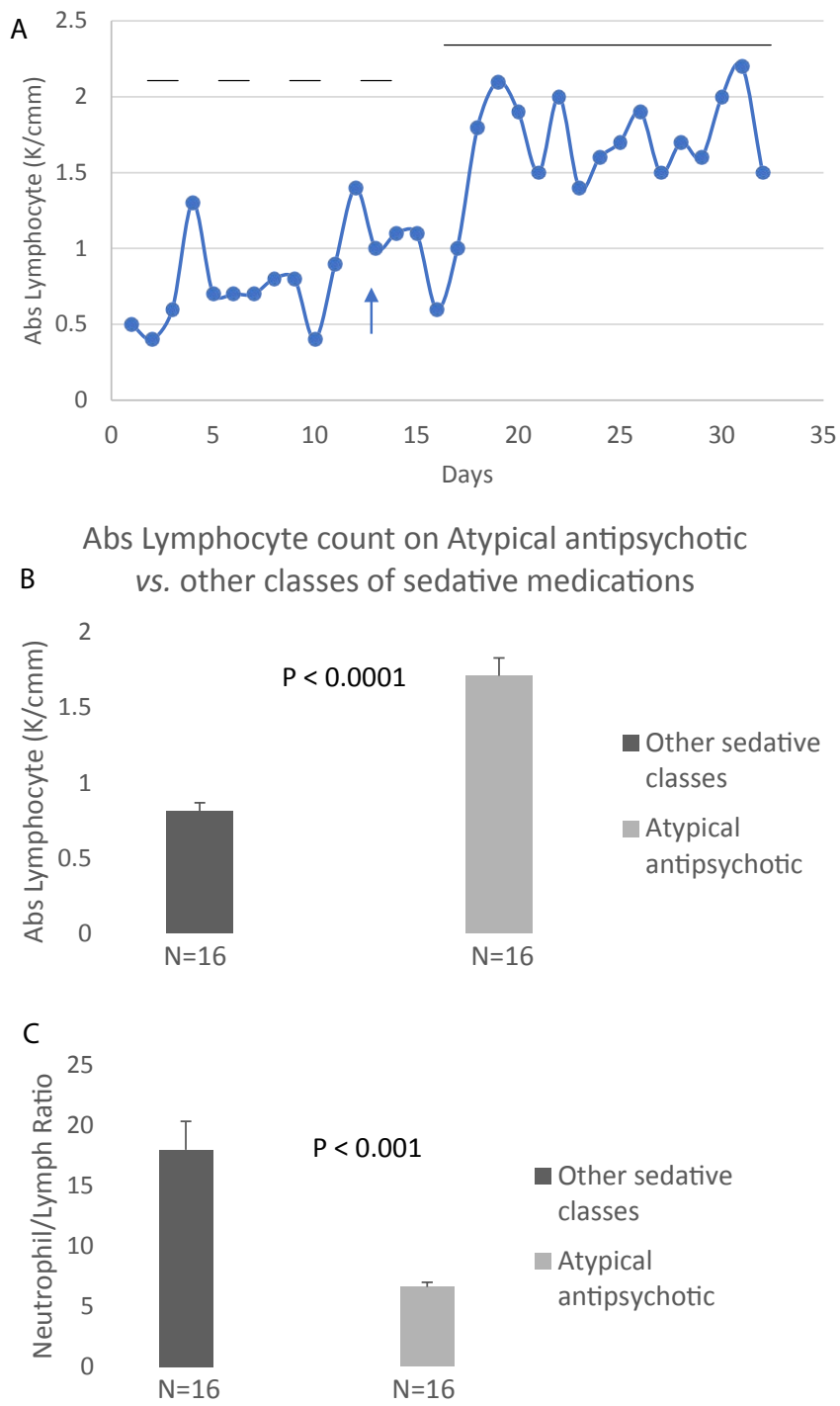

Figure 3: A 76-year old man with T2DM who developed hypoxemic respiratory failure and was intubated. A) During initial 15 days he was treated with a combination of D2 receptor blockers (Haldol) and benzodiazepines to control agitation/delirium in the ICU on the ventilator (dashed line). Following extubation (arrow) the drug regimen was changed to a nightly dose (for 18 consecutive days) of an oral SGA medication to control agitation/delirium (solid line). He was later discharged (in stable condition) from the hospital. B) The mean absolute lymphocyte count was significantly higher during a 16-day period on daily treatment with an SGA compared to the mean level during a comparable, preceding 16-day period on intermittent $\mathrm{D} 2$ receptor antagonist and/or benzodiazepine medications. C) The mean neutrophil/lymphocyte ratio (NLR) was significantly lower (6.6 vs. 18.0; $\mathrm{P}<0.001$ ) during the period on treatment with an SGA (atypical antipsychotic medication) compared to the corresponding preceding period on D2R antagonist and/or benzodiazepine medications to control agitation and delirium.

between the use of these classes of medications (to control delirium and agitation in the ICU) and higher absolute lymphocyte count and lower NLR suggests a possible immunomodulatory role for 5-HT2AR antagonists. In a mouse model of autoimmune hepatitis, treatment with the 5-HT2AR blocking anti-depressant medication mirtazapine substantially lowered hepatic inflammation [8]. Other evidence derived from in vitro and animal studies [6,7,9] suggests that 5 -hydroxytryptamine $2 \mathrm{~A}$ receptor antagonism may mediate an anti-inflammatory effect in part by interfering with the elaboration of pro-inflammatory cytokines from innate immune cells, e.g. macrophages. Despite unexplained 
lymphopenia, T-cell activation is another characteristic immunologic feature in severe COVID-19 infection [4]. The 5-hydroxytryptamine 2A receptor is widely expressed on peripheral immune cells (B cells, $\mathrm{T}$ cells) and vascular cells and among the diverse reported effects of 5-HT and/ or the 5-HT2A receptor are: T-cell activation [11] and increases in proinflammatory cytokines IL-6, interferon-gamma, and interleukin-2 [7,8].

Second-generation, atypical antipsychotic medications, tricyclic antidepressants and other antidepressant medications useful in treatment-resistant depression (trazadone, mirtazapine) all share high affinity for the 5-HT2A receptor. Several of the SGA medications also target the D2 dopamine receptor, the $\mathrm{H} 1$ histamine receptor, the alpha-1 adrenergic receptor, and the muscarinic acetylcholine receptor-making it difficult to ascribe a putative beneficial effect solely to actions at the 5-HT2A receptor. Yet (in our study) even administration of low doses of the antidepressant trazadone (relatively selective for 5-HT2AR antagonist activity) appeared to significantly upregulate lymphocyte count and downregulate the NLR within a time period (7-8 hours) consistent with the half-life of the medication.

Many of the patients in the present study having co-morbid psychiatric illness were treated (before and during COVID-19 infection) with an SGA or anti-depressant medication having serotonin $2 \mathrm{~A}$ receptor antagonist properties. These patients tended to experience a less severe form of illness in which the baseline NLR was generally $<10$. Mean baseline NLR did not differ significantly between patients who were treated or not treated with psychotropic medication having serotonin $2 \mathrm{~A}$ receptor antagonist properties. In our multi-variate logistic regression model that included adjustment for baseline NLR, 5-HT2AR antagonist medication use (vs.. non-use) was still a significant predictor of a lower risk of mortality. Taken together, there did not appear to be a selection bias toward use of 5-HT2AR antagonist medications in patients with less severe form of COVID-19 infection.

The present retrospective study was not designed to test for a possible causal relationship between the use of 5-HT2AR antagonistlike medications and mortality in severe COVID-19 infection. There are caveats important in the interpretation of the present findings: first, patients having co-morbid schizophrenia or major depression were generally younger compared to the overall patient cohort and second, certain genetic and/or environmental factors (including unknown immune factors) previously associated with an increased risk of psychiatric disorders may have independently affected mortality risk in COVID-19 pneumonia.

The study has several limitations. It was small and the results may only apply to the experience of older adult men having a number of different co-morbidities (diabetes, advanced age, hypertension, cardiovascular disease) previously associated with a higher risk of death in COVID-19 infection. More study in a larger group of patients (including women and patients having fewer co-morbidities) is needed to determine the generalizability of the findings.

In summary, advanced age and the non-use of certain antipsychotic and anti-depressant medications having shared antagonist activity on the 5-HT2A receptor was each a significant predictor of an increased risk of death in a small cohort of hospitalized, older adults who experienced COVID-19 infection requiring hospitalization. A future randomized trial may help determine whether an apparent association between the use of these classes of 'anti-inflammatory' medications and improved immunological parameters may translate into lower COVID-19 mortality in a subset of severely-affected patients.

\section{Acknowledgements}

We thank the dedicated and caring nurses, physicians, hospital workers and administrators at the Veterans Affairs New Jersey Healthcare System for their tireless and compassionate efforts on the part of the veterans and other patients affected by the COVID-19 pandemic. The views expressed here are solely those of the authors and do not represent the official position of the U.S. Department of Veterans Affairs or the US Government.

\section{References}

1. CDC, National Diabetes Fact Sheet (2011) Centers for Disease Control and Prevention [crossref].

2. Richardson S, Hirsch JS, Narasimhan M, et al. (2020) Presenting Characteristics, Comorbidities, and Outcomes Among 5700 Patients Hospitalized With COVID-19 in the New York City Area. JAMA. [crossref]

3. Selim A, Fincke G, Ren X, et al. (2004) Comorbidity Assessments Based on Patient Report: Results From the Veterans Health Study, Journal of Ambulatory Care Management 27(3): 281-295. [crossref]

4. Buszko, M, Park, J, Verthelyi, D. et al. (2020) The dynamic changes in cytokine responses in COVID-19: a snapshot of the current state of knowledge. Nat Immunol. [crossref]

5. Duerschmied D, Suidan GL, Demers M, et al. (2013) Platelet serotonin promotes the recruitment of neutrophils to sites of acute inflammation in mice. Blood 121(6): 1008-15. [crossref]

6. Herr N, Bode C, Duerschmied D, et al. (2017) The effects of Serotonin in immune Cells. Front Cardiovas Med [crossref]

7. Ding WM, Wang L, Han D, Gao J Pujun (2020) Serotonin: A Potent Immune Cell Modulator in Autoimmune Diseases. Frontiers in Immunology. [crossref]

8. Almishri W, Shaheen AA, Sharkey KA, Swain MG, et al. (2019) The antidepressant mirtazapine inhibits hepatic innate immune networks to attenuate immunemediated liver injury in mice Front Immunol 10: 803. [crossref]

9. Xiao J, Shao L, Shen J, Jiang W, Feng Y, Zheng P, Liu F, et al. (2016) Effects of ketanserin on experimental colitis in mice and macrophage function Int $\mathrm{J} \mathrm{Mol} \mathrm{Med}$ 37(3): 659-668. [crossref]

10. Liu J, Li S, Liu J, et al. (2020) Longitudinal characteristics of lymphocyte responses and cytokine profiles in the peripheral blood of SARS-CoV-2 infected patients EBioMedicine 55: 102763. [crossref]

11. Inoue $\mathrm{M}$, Okazaki $\mathrm{T}$, Kitazono $\mathrm{T}$, Mizushima $\mathrm{M}$, Omata $\mathrm{M}$, Ozaki S. et al (2011) Regulation of antigen-specific CTL and Th1 cell activation through 5-Hydroxytryptamine 2A receptor. Int Immunopharmacol 11(1): 67-73. [crossref]

\section{Citation:}

Mark B. Zimering, Tanzila Razzaki, Tiffany Tsang, John J. Shin (2020) Inverse Association between Serotonin 2A Receptor Antagonist Medication Use and Mortality in Severe COVID-19 Infection. Endocrinol Diabetes Metab J Volume 4(4): 1-5. 